Correspondence

Kiyoyasu Fukushima

kiyofuku@isahaya.jrc.or.jp

Received 3 December 2007

Accepted 8 February 2008

\section{A novel method for rapid detection of Streptococcus pneumoniae antigen in sputum and its application in adult respiratory tract infections}

\author{
Naomi Ehara, ${ }^{1}$ Kiyoyasu Fukushima, ${ }^{1}$ Hiroshi Kakeya, ${ }^{2}$ Hiroshi Mukae, ${ }^{2}$ \\ Suguru Akamatsu, ${ }^{3}$ Akiko Kageyama, ${ }^{3}$ Atsushi Saito ${ }^{1}$ and Shigeru Kohno ${ }^{2}$ \\ ${ }^{1}$ Division of Respiratory Medicine, Japanese Red Cross Nagasaki Genbaku Isahaya Hospital, \\ Nagasaki 859-0497, Japan \\ ${ }^{2}$ Second Department of Internal Medicine, Nagasaki University School of Medicine, Nagasaki \\ 852-8501, Japan \\ ${ }^{3}$ Otsuka Pharmaceutical Co. Ltd, Tokyo 108-8242, Japan
}

\begin{abstract}
A highly sensitive immunochromatography test kit, ODK0501, was developed using specific polyclonal antibodies against the C-polysaccharide moiety of Streptococcus pneumoniae for the rapid detection of $S$. pneumoniae antigen in sputum samples. The clinical utility of ODK0501 for this detection was evaluated prospectively in 52 adult patients with respiratory infections and compared with that of a urinary antigen detection kit. Overall, 21 patients $(40.4 \%)$ showed positive results with ODK0501, compared with 16 patients $(30.8 \%)$ using the urinary antigen detection kit, and S. pneumoniae was cultured from 18 patients. ODK0501 and the urinary antigen detection kit exhibited a sensitivity of 94.4 and $55.6 \%(P<0.01)$, respectively, and a specificity of 88.2 and $82.4 \%$, respectively. Eleven of thirteen patients with conflicting results between the two test kits exhibited consistent results for sputum cultures. Moreover, eight out of nine patients positive for ODK0501 and negative for the urinary antigen detection kit were $S$. pneumoniae culture-positive, including five who exhibited phagocytosis, indicating $S$. pneumoniae as a causative agent of infection, in Gram staining of sputum samples. These results suggest that the ODK0501 direct sputum detection kit is more clinically useful than the urinary antigen detection kit in adult patients with respiratory infections.
\end{abstract}

\section{INTRODUCTION}

Streptococcus pneumoniae is the most commonly detected causative agent of community-acquired pneumonia (File, 2003; Ishida et al., 2004; Llor et al., 2006; Saito et al., 2006), which is well recognized as being potentially severe (Saito et al., 2006). Mortality from this disease is particularly high in infants and the elderly, suggesting the need for an early appropriate pathogen-oriented therapy with antibacterial drugs after rapid identification of the causative agent. However, bacterial culture, the 'gold standard' for detection of causative micro-organisms, requires several days to yield results. Hence, empirical therapy is generally initiated prior to the availability of culture results, particularly in institutions without skilled laboratory personnel. Broad-spectrum antibiotics have been used increasingly, leading to the consequent development of drug-resistant bacteria. A simple and rapid technique for detecting S. pneumoniae is thus desirable. A urinary antigen detection kit for detecting S. pneumoniae antigens in urine (Binax) is commercially available for rapid

Abbreviations: C-ps, C-polysaccharide; NALC, N-acetyl-L-cysteine. diagnosis and has been widely used in clinical practice. However, it is associated with significant problems such as a high rate of false-positive results in children (Navarro et al., 2004), lack of detection immediately after the onset of infection and long-term antigen-positive results regardless of treatment (Marcos et al., 2003; Murdoch et al., 2003).

Here, we developed a rapid antigen detection kit (ODK0501) that can detect S. pneumoniae antigen in sputum samples within $25 \mathrm{~min}$. This kit specifically detects the C-polysaccharide (C-ps) moiety of S. pneumoniae, a common antigen of $S$. pneumoniae strains. In addition, we report evaluations of the optimal processing of sputum samples, which directly reflect inflammation in the lower respiratory tract, and the clinical significance of ODK0501 for detecting $S$. pneumoniae antigen in sputum samples from adult patients with pneumococcal respiratory infections.

\section{METHODS}

Patient population. This study was approved by the Institutional Review Board of the Japanese Red Cross Nagasaki Genbaku Isahaya 
Hospital. All patients provided informed consent for sample collection. A total of 52 adult inpatients and outpatients with suspected respiratory infection ( 25 males, 27 females; median age 66.8 years; age range 23-95 years) was examined at the Japanese Red Cross Nagasaki Genbaku Isahaya Hospital from October 2005 to November 2006.

Methods and sample collection. Single sputum and urine samples were collected from patients at the time when a presumptive diagnosis of infection was made, and were refrigerated at $4{ }^{\circ} \mathrm{C}$ until analysis. The appearance of each sputum sample was assessed according to the classification scheme of Miller \& Jones (1963). Sputum sample quality was evaluated by assessing the number of inflammatory and epithelial cells; samples were considered viable if there were at least 10 epithelial cells and $>25$ polymorphonuclear leukocytes per microscope high-power field, in accordance with the criteria of Murray \& Washington (1975). Sputum samples were analysed by Gram staining, culture and the ODK0501 test kit, while the urine samples were assessed using the commercially available Binax NOW S. pneumoniae urinary antigen detection kit.

Principles of measurement with ODK0501. ODK0501 is an immunochromatography method for detecting C-ps, a common antigen of $S$. pneumoniae that has the same structure in all $S$. pneumoniae strains regardless of serotype (Skov Sorensen, 1995). Initially, application of samples onto the ODK0501 test kit through a dropping window causes a reaction of the C-ps in the samples with anti-pneumococcal C-ps colloidal gold-labelled rabbit polyclonal antibody forming an antigen-antibody complex. The resulting immunoconjugates are transferred via capillary action to the upper membrane and captured by the anti-pneumococcal C-ps solid-phase rabbit polyclonal antibodies, thereby forming sandwich conjugates in the sample. The eventual presence of a visually detectable red test line derived from colloidal gold particles on the membrane indicates the presence of C-ps. In contrast, anti-pneumococcal C-ps rabbit polyclonal antibodies that are not bound to C-ps passed through the test line and are captured by solid-phase goat anti-rabbit IgG antibodies on the control line, forming a red line (Fig. 1).

Measurement and interpretation of results obtained with ODK0501. A swab used to collect sputum was placed in a tube containing a sample extract solution; the tube was then shaken with the swab tip, with the tube pinched around the top. After leaving the tube to stand for $5 \mathrm{~min}$, the swab tip was removed while squeezing the tube. Next, a filter was mounted on the tube and the extract was dropped onto the test kit. Approximately $20 \mathrm{~min}$ later, the results were assessed; a positive test result was indicated by the presence of the red test and control lines, whereas a negative test result was indicated by the presence of only the control line.

Measurement and interpretation of results obtained with the urinary antigen detection kit. These were conducted in accordance with the manufacturer's guidelines (Dominguez et al., 2001).

Culture techniques. Gram staining was performed using conventional methods, following incubation of cultures at $37{ }^{\circ} \mathrm{C}$ for $24 \mathrm{~h}$ on blood and chocolate agar. After incubation, the presumptive colonies of S. pneumoniae were picked and identified.

Determination of the limit of detection. The limit of detection of ODK0501 was determined using purified C-ps, due to differences in bacterial loads among S. pneumoniae cultures. A C-ps solution was

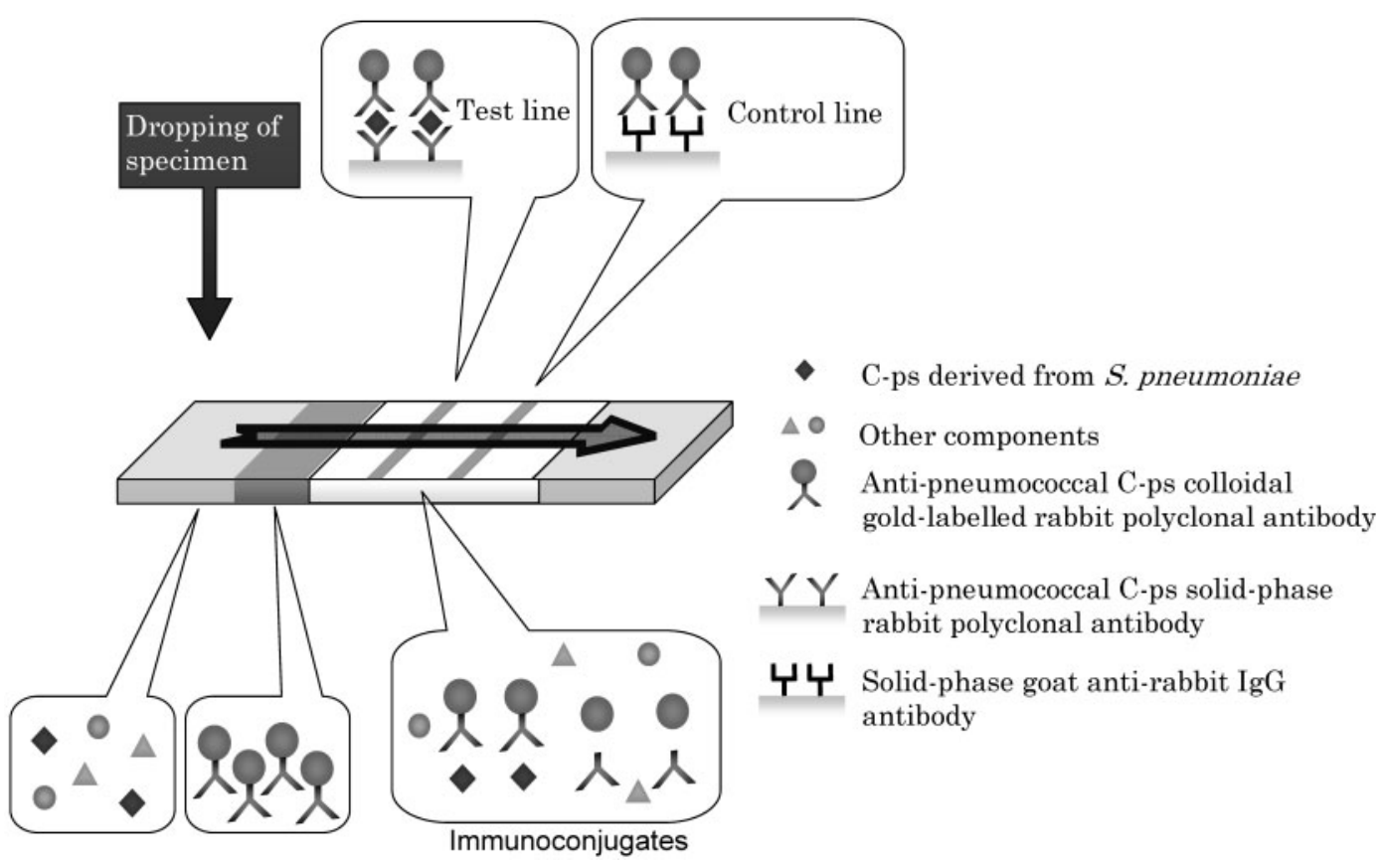

Fig. 1. Principles of measurement with the ODK0501 sputum antigen detection test kit. Application of an extract of sputum samples onto the ODK0501 kit through a dropping window causes a reaction of S. pneumoniae antigen (C-ps) with antipneumococcal C-ps polyclonal antibodies. The resulting immunoconjugates are transferred to the upper membrane, and captured by the anti-pneumococcal C-ps solid-phase polyclonal antibodies, thereby forming sandwich conjugates in the sample. A positive test result is indicated by the presence of both a test and a control line, whereas a negative result is indicated by the appearance of only a single control line. 
prepared at $3.0 \mathrm{ng} \mathrm{ml}^{-1}$, and then diluted fourfold to $0.75 \mathrm{ng} \mathrm{ml}^{-1}$ and eightfold to $0.375 \mathrm{ng} \mathrm{ml}^{-1}$ using a sample extraction reagent. The three C-ps solutions were then measured using the sputum test kit and the presence or absence of lines was interpreted visually. A previous study confirmed that an extract of $0.75 \mathrm{ng} \mathrm{ml}^{-1}$ is equivalent to $1.3 \times 10^{4}$ c.f.u. S. pneumoniae ATCC $49619 \mathrm{ml}^{-1}$ (data not shown).

Confirmation of cross-reactivity. A total of 60 potential crossreactants, including 41 microbes (30 species) and 19 viruses (7 species) (Table 1), were measured using the ODK0501 kit, and the presence or absence of cross-reactivity was interpreted visually.

Examination of methods for sputum sample collection: effects of solvents on detection of $\boldsymbol{S}$. pneumoniae antigen in sputum. Prior to examination of the methods for sputum sample collection, the effects of solvents on the detection of S. pneumoniae antigen in sputum were assessed using pneumococcal cultures. A pneumococcal suspension was prepared at a concentration of $6.5 \times 10^{6}$ c.f.u. $\mathrm{ml}^{-1}$ in PBS, and then mixed and prepared with various sputum solvents at the ratios indicated below. Each prepared solution was added to 25$500 \mu \mathrm{l}$ extraction reagent included in the kit and measured. The intensities of the lines obtained were determined using a densitometer for quantitative evaluation of the effects of the solvents. Sputum solvents were processed using four methods. In the first method, $200 \mu \mathrm{l}$ Sputazyme (Kyokuto Pharmaceutical) was mixed with $100 \mu \mathrm{l}$ pneumococcal suspension and the mixture was left to stand for $15 \mathrm{~min}$ at room temperature. In the second method, $200 \mu \mathrm{l}$ sodium citrate at a final concentration of $50 \mathrm{mM}, 0.5 \mathrm{M} \mathrm{NaOH}$ and $31 \mathrm{mM}$ $\mathrm{N}$-acetyl-L-cysteine (NALC) (Wako Pharmaceutical) were mixed with $100 \mu \mathrm{l}$ pneumococcal suspension. In the third method, $200 \mu \mathrm{l}$ NALC at a final concentration of $31 \mathrm{mM}$ prepared with saline solution was mixed with $100 \mu$ pneumococcal suspension. In the fourth method, $200 \mu \mathrm{l}$ phosphate buffer solution was added to $100 \mu \mathrm{l}$ of the directly collected sputum culture diluent and the mixture was suspended.

Examination of methods for sputum sample collection: evaluation of sputum. The Sputazyme method described above was compared with the direct collection method using four different sputum samples. Sputazyme solution was prepared in equal, twofold and threefold quantities relative to the sputum and then assessed. The direct collection method involved directly collecting the sputum samples using the swabs included in the kit.

Statistical analysis. Comparison of the sensitivity and specificity of ODK0501 and the urinary antigen detection kit were performed using Fisher's exact probability test. Nominal values of $P<0.05$ were considered statistically significant.

\section{RESULTS AND DISCUSSION}

The newly developed test kit ODK0501 is non-invasive, as sputum is used as the test sample. ODK0501 detects a common antigen of all types of $S$. pneumoniae, regardless of the capsule serotype.

\section{Limit of detection and cross-reactivity}

Positive results were observed in all sample extracts at C-ps concentrations of 3.0, 0.75 and $0.375 \mathrm{ng} \mathrm{ml}^{-1}$. However, as the coloured lines of weak intensity obtained with the extracts at $0.375 \mathrm{ng} \mathrm{ml}^{-1}$ caused difficulty in the interpretation of the results, the limit of detection of ODK0501 was considered to be $0.75 \mathrm{ng} \mathrm{ml}^{-1}$. The urinary antigen detection kits detected the extracts at C-ps concentrations of $0.375 \mathrm{ng} \mathrm{ml} \mathrm{m}^{-1}$, the same as the ODK0501 kit (data not shown). Cross-reactivity was assessed towards various sample extracts from 41 microbes (30 species) other than S. pneumoniae and 19 viral strains (7 species) including major causative agents and viruses of respiratory infections such as intraoral microbes. Cross-

Table 1. Results of cross-reactivity tests

Numbers in parentheses indicate the number of strains examined.

\begin{tabular}{|llll|}
\hline Agent name & Cross-reactivity & \multicolumn{1}{c|}{ Agent name } & Cross-reactivity \\
\hline Bacteroides fragilis & No & Streptococcus constellatus (2) & No \\
Bordetella pertussis & No & Streptococcus equi & No \\
Candida albicans & No & Streptococcus intermedius & Yes \\
Corynebacterium pseudodiphtheriticum & No & Streptococcus mitis & No \\
Cryptococcus neoformans & No & Streptococcus oralis & No \\
Eubacterium limosum & No & Streptococcus sanguinis & No \\
Haemophilus influenzae (8) & No & Streptococcus sp. & No \\
Haemophilus parainfluenzae & No & Chlamydia trachomatis & No \\
Klebsiella pneumoniae & No & Chlamydophila pneumoniae & No \\
Legionella pneumophila (3) & No & Chlamydophila psittaci & No \\
Micromonas micros & Yes & Mycoplasma pneumoniae & No \\
Moraxella catarrhalis & No & Adenovirus (8) & No \\
Neisseria meningitidis & No & Coxsackievirus (2) & No \\
Prevotella intermedia & No & Echovirus (2) & No \\
Prevotella melaninogenica & No & No \\
Pseudomonas aeruginosa & No & No \\
Staphylococcus aureus (2) & No & Influenza B virus & \\
Streptococcus agalactiae & No & Parainfluenza virus (3) & \\
Streptococcus anginosus & No & Respiratory syncytial virus & \\
& & \\
\end{tabular}


reactivity was observed only for Micromonas micros ATCC $33270\left(8.0 \times 10^{6}\right.$ c.f.u. $\left.\mathrm{ml}^{-1}\right)$ and Streptococcus intermedius ATCC $9895\left(7.0 \times 10^{7}\right.$ c.f.u. $\left.\mathrm{ml}^{-1}\right)$. However, crossreactivity was not found for either $M$. micros ATCC 33270 in a half-concentration extract of $4.1 \times 10^{6}$ c.f.u. $\mathrm{ml}^{-1}$ or S. intermedius ATCC 9895 in a half-concentration extract of $3.5 \times 10^{7}$ c.f.u. $\mathrm{ml}^{-1}$, suggesting that crossreactivity may only be a problem in patients with high bacterial loads. No cross-reactivity was found for the remaining 39 microbes and 19 viral strains (Table 1). No cross-reactivity was detected for Streptococcus oralis or Streptococcus mitis, both of which are known to react with the Binax NOW urinary antigen detection kit for $S$. pneumoniae (Murdoch \& Reller, 2003). We consider that the difference in cross-reactivity of both kits is due to the characteristics of the antibodies rather than their sensitivity. It is also difficult to rule out the possibility of carriage.

\section{Examination of the processing of sputum samples}

S. pneumoniae antigens were detected with the Sputazyme, NALC/saline and direct collection processing methods, but not with the NALC/NaOH method (Fig. 2a). Moreover, comparison of the line intensities among the three methods indicated that the Sputazyme method (including centrifugal processing) and direct collection method were optimal for processing. A comparison of the Sputazyme method with the direct collection method using four different sputum samples is shown in Fig. 2(b). Three different sputum samples (samples 2-4) exhibited almost the same line intensities with both methods, whereas a lower line intensity was obtained for sputum sample 1 with the direct collection method. Thus, considering the rapidity and simplicity of processing, direct collection of purulent sputum from patients using a swab, based on the guidelines for the use of ODK0501, appeared to be the optimal method. The present study confirmed that, in addition to direct sample collection of purulent sputum using a swab, other sampling methods involving dissolution of specimens in sputum solvents are also appropriate for assessment using ODK0501. The antigen-antibody reaction may be inhibited by $\mathrm{NaOH}$ in the $\mathrm{NALC} / \mathrm{NaOH}$ method, indicating that this method cannot be used for sample processing with ODK0501. On the other hand, specimens processed with the Sputazyme method frequently used for sputum cultures yielded acceptable results.

\section{Comparison of the sensitivities and specificities between ODK0501 and the urinary antigen detection kit}

The 52 patients were clinically diagnosed with respiratory infections such as acute bronchitis $(n=6)$, acute exacerbation of chronic respiratory tract infection $(n=14)$ and pneumonia $(n=32)$. S. pneumoniae culture-positive patients included 4 with acute exacerbation of chronic respiratory tract infection $(28.6 \%)$ and 14 with pneumonia $(43.8 \%)$. In these patients, based on sputum culture

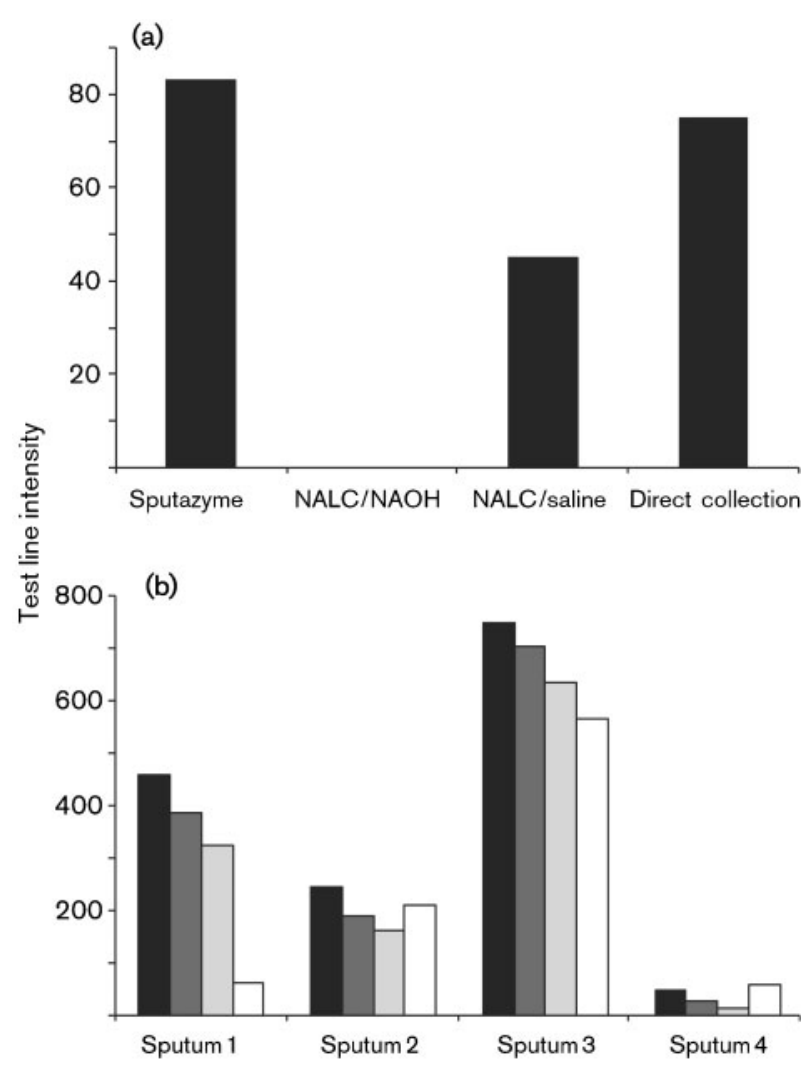

Fig. 2. Effects of sputum solvents. (a) Evaluation of various solvents following culture. (b) Effects of solvents on diluted sputum were evaluated using four different sputum samples: black bars, Sputazyme at an equal quantity relative to sputum; dark-grey bars, Sputazyme at a twofold quantity relative to sputum; light-grey bars, Sputazyme at a threefold quantity relative to sputum; white bars, direct collection using a swab.

results, ODK0501 exhibited a high sensitivity of $94.4 \%$ (17/18) and a specificity of $88.2 \%$ (30/34), whereas the urinary antigen detection kit exhibited a sensitivity of $55.6 \%(10 / 18)$ and a specificity of $82.4 \%$ (28/34) (Table 2). Thus, the two tests produced almost the same specificity, but the sensitivity of the urinary antigen test kit was

Table 2. Sensitivity and specificity of ODK0501 and the urinary antigen detection kit for respiratory tract infections $(n=52)$

\begin{tabular}{|llrcc|}
\hline \multirow{2}{*}{ Kit } & & \multicolumn{3}{c|}{ Sputum culture } \\
\cline { 3 - 5 } & & Positive & Negative & Total \\
\hline ODK0501 & Positive & 17 & 4 & 21 \\
& Negative & 1 & 30 & 31 \\
Urinary antigen & Positive & 10 & 6 & 16 \\
detection kit & Negative & 8 & 28 & 36 \\
& Total & 18 & 34 & 52 \\
\hline
\end{tabular}


approximately $40 \%$ lower than that of ODK0501. Comparisons of the sensitivities and specificities between the two test kits revealed a statistically significant difference in the sensitivity $(P<0.01$, two-tailed Fisher's exact probability test).

We developed and evaluated the new ODK0501 detection kit for S. pneumoniae antigens in sputum. Data regarding the possibility of false-positive carriage results are very important. However, the incidence of pneumococcal carriage in adults is very rare, e.g. $4 \%$ in nasopharyngeal and/or oropharyngeal samples (Lieberman et al., 2006). In some elderly individuals, infants and children, asymptomatic carriage of $S$. pneumoniae can be detected in the nasal cavity, and the nasopharyngeal and intraoral regions (Abe et al., 2001; Hamer et al., 2002). In this study, we evaluated only adult patients with respiratory tract infections, because of the difficulty of collecting sputum in children.

Among 13 adult patients with conflicting results for the 2 tests, 9 had positive results with ODK0501 and negative results with the urinary antigen detection kit, 8 of whom were $S$. pneumoniae culture-positive, including 5 who exhibited phagocytosis of Gram-positive bacteria after Gram staining. In contrast, four patients exhibited negative results with ODK0501 and positive results with the urinary antigen detection kit, and only one of these patients was $S$. pneumoniae culture-positive with no phagocytosis of Gram-positive bacteria (Table 3). Of the 12 patients who were S. pneumoniae culture-positive with phagocytosis of Gram-positive bacteria, all were positive using the ODK0501 kit, whereas only 8 patients were positive using the urinary antigen detection kit. Thus, the ODK0501 direct sputum detection kit can discriminate the causative agent from its indigenous colonies in adult patients with $S$. pneumoniae infection. The false-negative results for urinary antigen detection in at least five patients who were $S$. pneumoniae culture-positive with phagocytosis of Grampositive bacteria represent a significant problem for the urinary antigen detection kit. ODK0501 could sensitively detect C-ps antigen in $S$. pneumoniae culture-positive sputum specimens. ODK0501 may thus be useful for a clinically rapid and supportive diagnosis of pneumococcal infection in combination with clinical symptoms and the appearance of sputum.

Detection of C-ps in sputum samples using ELISA has been reported in studies dating back to the late 1980s through to the early 1990s (Krook \& Holmberg, 1987; Burman et al., 1991). Although ELISA has a high sensitivity, it is complex and requires considerable skill. Moreover, the extended time required for the assay hampers its routine use in the clinical management of critical pneumonia infections, such as those caused by S. pneumoniae or Legionella pneumoniae. Rapid methods using an immunochromatography detection kit for S. pneumoniae or L. pneumoniae antigens in urine samples have been developed more recently (Dominguez et al., 2001). We consider that using the detection kit ODK0501 for analysing sputum samples obtained from local sites of infection will be extremely useful for detecting the causative $S$. pneumoniae in respiratory infections.

In conclusion, we have described here a test kit for rapid detection of antigen from $S$. pneumoniae, the most common causative agent of pneumonia, in sputum samples, and its application to the diagnosis of respiratory infections. S. pneumoniae is a Gram-positive diplococcus, whose surface is covered by a cell wall surrounded by a capsule, which provides strong resistance to bactericidal phagocytosis. The capsule consists of a capsular polysaccharide with various structures (Skov Sorensen, 1995) and

Table 3. List of conflicting results for ODK0501 and the urinary antigen detection kit

\begin{tabular}{|c|c|c|c|}
\hline $\begin{array}{c}\text { Sputum S. pneumoniae } \\
\text { antigen }\end{array}$ & $\begin{array}{c}\text { Urinary } S . \text { pneumoniae } \\
\text { antigen }\end{array}$ & Sputum culture & Gram staining (GPC phagocytosis)* \\
\hline Positive & Negative & S. pneumoniae $3+$ & $3+($ phagocytosis $)$ \\
\hline Positive & Negative & S. pneumoniae $2+$ & $2+$ (phagocytosis $)$ \\
\hline Positive & Negative & S. pneumoniae $2+$ & $2+$ (no phagocytosis) \\
\hline Positive & Negative & S. pneumoniae $3+$ & $3+$ (phagocytosis $)$ \\
\hline Positive & Negative & S. pneumoniae $3+$ & $2+$ (phagocytosis $)$ \\
\hline Positive & Negative & S. pneumoniae $3+$ & $3+$ (no phagocytosis) \\
\hline Positive & Negative & S. pneumoniae $3+$ & $2+$ (no phagocytosis) \\
\hline Positive & Negative & S. pneumoniae $3+$ & $3+$ (phagocytosis $)$ \\
\hline Positive & Negative & H. influenzae $3+$ & $1+$ (no phagocytosis) \\
\hline Negative & Positive & S. pneumoniae $2+$ & $2+$ (no phagocytosis) \\
\hline Negative & Positive & Haemophilus sp. \pm & $2+$ (no phagocytosis) \\
\hline Negative & Positive & Normal flora & $2+$ (no phagocytosis) \\
\hline Negative & Positive & Normal flora & $3+$ (no phagocytosis) \\
\hline
\end{tabular}

GPC, Gram-positive cocci.

${ }^{\star}$ Bold text indicates the five patient samples where phagocytosis was observed. 
its serotypes are classified into 90 subtypes according to the type of capsule (Henrichsen, 1995). As previous tests conducted to detect capsular polysaccharide have been associated with problems, such as lack of coverage of all serotypes and insufficient sensitivity of detection (Harding et al., 1979; Leeming et al., 2005), a new assay with high sensitivity that is rapid and easy to perform is needed. The urinary antigen detection kit based on immunochromatography analysis has been used as a rapid diagnostic test for $S$. pneumoniae. Its principle of measuring a common antigen of $S$. pneumoniae enables all types of $S$. pneumoniae to be detected, regardless of capsule serotype. In addition, the test kit is non-invasive, as urine is used as the test sample, and it has therefore been widely used in clinical practice. However, it has been associated with problems including false-positive results in children with a high likelihood of detection of indigenous $S$. pneumoniae (Dowell et al., 2001), detection of S. pneumoniae antigens for approximately 1-3 months in some patients even after treatment (Marcos et al., 2003; Murdoch et al., 2003a) and difficulty in determining whether positive test results are due to true infection or a reflection of colonization in some patients. Furthermore, the test has been reported to exhibit a variable but relatively low sensitivity of $50-80 \%$ (Dominguez et al., 2001; Gutiérrez et al., 2003; Murdoch et al., 2001), leading to a need for a further increase in the test sensitivity.

In adult patients with $S$. pneumoniae infection, the newly developed test kit ODK0501 can immediately provide highly sensitive results, demonstrating the presence of S. pneumoniae in sputum, as sputum derived from a local region of infection is used as the test sample. We suggest that this test will be very useful both for rapid diagnosis with differentiation from indigenous agents and for determining the choice of appropriate antimicrobial agents for treatment of respiratory tract infections in clinical practice. Future studies will be necessary to compare ODK0501 results using sputum samples from individuals who also test positive for nasopharyngeal carriage of $S$. pneumoniae with those who test negative for pneumococcal carriage.

\section{ACKNOWLEDGEMENTS}

The authors thank Mr Toshinori Sagara for his skilful technical assistance.

\section{REFERENCES}

Abe, S., Ishihara, K. \& Okuda, K. (2001). Prevalence of potential respiratory pathogens in the mouths of elderly patients and effects of professional oral care. Arch Gerontol Geriatr 32, 45-55.

Burman, L. A., Trollfer, B., Andersson, B., Henrichsen, J., Juto, P., Kallings, I., Lagergård, T., Möllby, R. \& Norrby, R. (1991). Diagnosis of pneumonia by cultures, bacterial and viral antigen detection tests, and serology with special reference to antibodies against pneumococcal antigens. J Infect Dis 163, 1087-1093.
Dominguez, J., Gali, N., Blanco, S., Pedroso, P., Prat, C., Matas, L. \& Ausina, V. (2001). Detection of Streptococcus pneumoniae antigen by a rapid immunochromatographic assay in urine samples. Chest 119, 243-249.

Dowell, S. F., Garman, R. L., Liu, G., Levine, O. S. \& Yang, Y. H. (2001). Evaluation of Binax NOW, an assay for the detection of pneumococcal antigen in urine samples, performed among pediatric patients. Clin Infect Dis 32, 824-825.

File, T. M., Jr (2003). Community-acquired pneumonia. Lancet 362, 1991-2001.

Gutiérrez, F., Masia, M., Rodriguez, J. C., Ayelo, A., Soldan, B., Cebrian, L., Mirete, C., Royo, G. \& Hidalgo, A. M. (2003). Evaluation of the immunochromatographic Binax NOW assay for detection of Streptococcus pneumoniae urinary antigen in a prospective study of community-acquired pneumonia in Spain. Clin Infect Dis 36, 286-292.

Hamer, D. H., Egas, J., Estrella, B., MacLeod, W. B., Griffiths, J. K. \& Semoertegui, F. (2002). Assessment of the Binax NOW Streptococcus pneumoniae urinary antigen test in children with nasopharyngeal pneumococcal carriage. Clin Infect Dis 34, 1025-1028.

Harding, S. A., Scheld, W. M., McGowan, M. D. \& Sande, M. A. (1979). Enzyme-linked immunosorbent assay for detection of Streptococcus pneumoniae antigen. J Clin Microbiol 10, 339-342.

Henrichsen, J. (1995). Six newly recognized types of Streptococcus pneumoniae. J Clin Microbiol 33, 2759-2762.

Ishida, T., Hashimoto, T., Arita, M., Tojo, Y., Tachibana, H. \& Jinnai, M. (2004). A 3-year prospective study of a urinary antigen-detection test for Streptococcus pneumoniae in community-acquired pneumonia: utility and clinical impact on the reported etiology. J Infect Chemother 10, 359-363.

Krook, A. \& Holmberg, H. (1987). Pneumococcal antigens in sputa: ELISA for the detection of pneumococcal C-polysaccharide in sputa from pneumonia patients. Diagn Microbiol Infect Dis 7, 73-75.

Leeming, J. P., Cartwright, K., Morris, R., Martin, S. A. \& Smith, M. D.on behalf of the South-West Pneumococcus Study Group (2005). Diagnosis of invasive pneumococcal infection by serotypespecific urinary antigen detection. J Clin Microbiol 43, 4972-4976.

Lieberman, D., Shleyfer, E., Castle, H., Terry, A., Harman-Boehm, I., Delgado, J., Peled, N. \& Lieberman, D. (2006). Nasopharyngeal versus oropharyngeal sampling for isolation of potential respiratory pathogens in adults. J Clin Microbiol 44, 525-528.

Llor, C., Cots, J. M. \& Herreras, A. (2006). Bacterial etiology of chronic bronchitis exacerbations treated by primary care physicians. Arch Bronconeumol 42, 388-393.

Marcos, M. A., Jimenez de Anta, M. T., de la Bellacasa, J. P., González, J., Martínez, E., García, E., Mensa, J., de Roux, A. \& Torres, A. (2003). Rapid urinary antigen test for diagnosis of pneumococcal community-acquired pneumonia in adults. Eur Respir J 21, 209-214.

Miller, D. L. \& Jones, R. (1963). A study of techniques for the examination of sputum in a field survey of chronic bronchitis. Am Rev Respir Dis 88, 473-483.

Murdoch, D. R. \& Reller, L. B. (2003). Immunochromatographic test for rapid detection of Streptococcus pneumoniae in the nasopharynx. J Clin Microbiol 41, 2271.

Murdoch, D. R., Laing, R. T., Mills, G. D., Karalus, N. C., Town, G. I., Mirrett, S. \& Reller, L. B. (2001). Evaluation of a rapid immunochromatographic test for detection of Streptococcus pneumoniae antigen in urine samples from adults with community-acquired pneumonia. $J$ Clin Microbiol 39, 3495-3498.

Murdoch, D. R., Laing, R. T. \& Cook, J. M. (2003). The NOW Streptococcus pneumoniae urinary antigen test positivity rate 6 weeks 
after pneumonia onset and among patients with COPD. Clin Infect Dis 37, 153-154.

Murray, P. R. \& Washington, J. A. (1975). Microscopic and bacteriologic analysis of expectorated sputum. Mayo Clin Proc 50, 339.

Navarro, D., Garcia, M. L., Gimeno, C., Escribano, A. \& Garcia, L. (2004). Performance of the Binax NOW Streptococcus pneumoniae urinary antigen assay for diagnosis of pneumonia in children with underlying pulmonary diseases in the absence of acute pneumococcal infection. J Clin Microbiol 42, 4853-4855.

Saito, A., Kohno, S., Matsushima, T., Watanabe, A., Oizumi, K., Yamaguchi, K., Oda, H. \& the Study Group (2006). Prospective multicenter study of the causative organisms of community-acquired pneumonia in adults in Japan. J Infect Chemother 12, 63-69.

Sorensen, U. B. (1995). Pneumococcal polysaccharide antigens: capsules and C-polysaccharide. Dan Med Bull 42, 47-53. 